\title{
Nitric Oxide Mediated Degradation of CYP2A6 via the Ubiquitin-Proteasome Pathway in Human Hepatoma Cells ${ }^{\text {[ }}$
}

\author{
John Cerrone, Jr., Choon-myung Lee, Tian Mi, and @Edward T. Morgan \\ Department of Pharmacology and Chemical Biology, Emory University, Atlanta, Georgia
}

Received November 7, 2019; accepted April 6, 2020

\begin{abstract}
Several cytochrome P450 enzymes are known to be down-regulated by nitric oxide (NO). CYP2A6 is responsible for the metabolism of nicotine and several other xenobiotics, but its susceptibility to downregulation by NO has not been reported. To address this question, we used Huh7 human hepatoma cell lines to express CYP2A6 with a C-terminal V5 tag (CYP2A6V5). NO donor treatment [dipropylenetriamine NONOate (DPTA)] down-regulated CYP2A6 protein to approximately $40 \%$ of control levels in 4 hours. An NO scavenging agent protected CYP2A6 from down-regulation by DPTA in a concentration-dependent manner, demonstrating that the downregulation is NO-dependent. Experiments with the protein synthesis inhibitor cycloheximide showed that CYP2A6 protein downregulation occurs posttranslationally. In the presence of proteasome inhibitors MG132 or bortezomib, NO-treated cells showed an accumulation of a high molecular mass signal, whereas autophagy inhibitors chloroquine and 3-methyladenine and the lysosomal and calpain inhibitor E64d had no effect. Immunoprecipitation of CYP2A6 followed by Western blotting with an antiubiquitin antibody showed that the high molecular mass species contain polyubiquitinated
\end{abstract}

CYP2A6 protein. This suggests that NO led to the degradation of protein via the ubiquitin-proteasome pathway. The down-regulation by NO was blocked by the reversible CYP2A6 inhibitor pilocarpine but not by the suicide inhibitor methoxsalen, demonstrating that down-regulation requires $\mathrm{NO}$ access to the active site but does not require catalytic activity of the enzyme. These findings provide novel insights toward the regulation of CYP2A6 in a human cell line and can influence our understanding of CYP2A6-related drug metabolism.

\section{SIGNIFICANCE STATEMENT}

This study demonstrates that the nicotine metabolizing enzyme CYP2A6 is down-regulated by nitric oxide, a molecule produced in large amounts in the context of inflammation and that is also inhaled from cigarette smoke. This occurs via ubiquitination and proteasomal degradation, and does not require catalytic activity of the enzyme. This work adds to the growing knowledge of the selective effect and mechanism of action of nitric oxide (NO) on cytochrome P450 enzymes and suggests a possible novel mode of interaction between nicotine and NO in cigarette smokers.

\section{Introduction}

Nitric oxide (NO) is a free radical gas involved in numerous biologic processes, including vasodilation, neuronal signaling, and immune function (Park et al., 2017a). NO levels are elevated by various pharmaceuticals that directly release $\mathrm{NO}$ or via induction of endogenous nitric oxide synthases (Laufs and Liao, 1998; Agvald et al., 2002; Antoniades et al., 2011). NO, acting as a diffusible signaling molecule across cell membranes, interacts directly with proteins, affecting protein turnover and activity (Kim et al., 2004; Hess and Stamler, 2012). Furthermore, NO reacts with oxygen and reactive oxygen species to form reactive nitrogen species that can modify proteins to regulate their function or expression (Radi, 2018).

There are three major mechanisms by which reactive nitrogen species modify proteins: heme nitrosylation, tyrosine nitration, and protein S-nitrosylation (Bartesaghi and Radi, 2018). In soluble guanylyl cyclase

This work was supported by the National Institutes of Health Institute of General Medical Science [Grant R01 GM 069971]

https://doi.org/10.1124/dmd.119.089961.

SThis article has supplemental material available at dmd.aspetjournals.org.
(sGC), NO binding to heme induces cGMP formation and activation of protein kinase G (Arnold et al., 1977; Hunt and Lehnert, 2015; Beuve, 2017; Shah et al., 2018). sGC cysteine residues are also a target for NO, and S-nitrosylation resulted in decreased responsiveness of sGC (Sayed et al., 2007; Shah et al., 2018). S-nitrosylation has been equated to protein phosphorylation in cell signaling pathways; however, in some cases, aberrant S-nitrosylation of protein can lead to protein misfolding contributing to pathogenesis of various diseases including Alzheimer's, Parkinson's, and Huntington's (Zahid et al., 2014; Nakamura et al., 2015; Zhao et al., 2015). Tyrosine nitration is implicated in a wide array of pathogeneses including lung carcinogenesis and metastasis and a number of age-related diseases including Alzheimer's and Parkinson's (Radi, 2013; Yeo et al., 2015; Zhan et al., 2018). Furthermore, tyrosine nitration has been associated with physiologic aging, and nitration of transmembrane peptides has been connected to lipid peroxidation, a hallmark of tissue degeneration (Mylonas and Kouretas, 1999; Bartesaghi et al., 2017; Chakravarti and Chakravarti, 2017).

One family of proteins affected by reactive nitrogen species are the cytochrome P450 (P450) proteins. These enzymes metabolize many xenobiotics and are responsible for many biosynthetic pathways pivotal

ABBREVIATIONS: 3MA, 3-methyladenine; DMEM, Dulbecco's modified Eagle's medium; DPTA, dipropylenetriamine NONOate; E64d, (2S,3S)trans-Epoxysuccinyl-L-leucylamido-3-methylbutane ethyl ester; GAPDH, glyceraldehyde-3-phosphate dehydrogenase; GSNO, S-nitrosoglutathione; HMM, high molecular mass; MG132, carbobenzoxy-L-leucyl-L-leucyl-L-leucinal; NO, nitric oxide; P450, cytochrome P450; PTIO Carboxy PTIO, , 2-(4-carboxyphenyl)-4,5-dihydro-4,4,5,5-tetramethyl-1H-imidazolyl-1-oxy-3-oxide, monopotassium salt; sGC, soluble guanylyl cyclase; $\mathrm{SIN}-1$, 3-morpholinosydnonimine; SNAP, S-nitroso-N-acetyl-DL-penicillamine. 
A

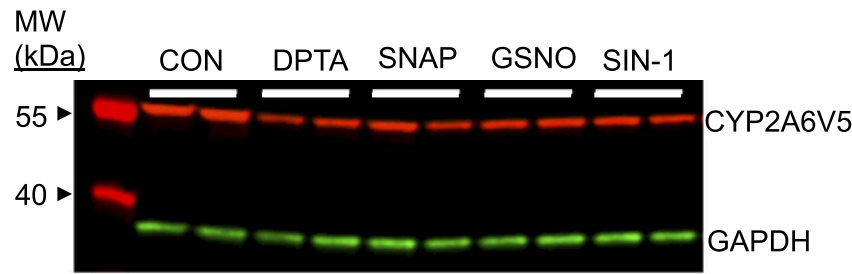

B

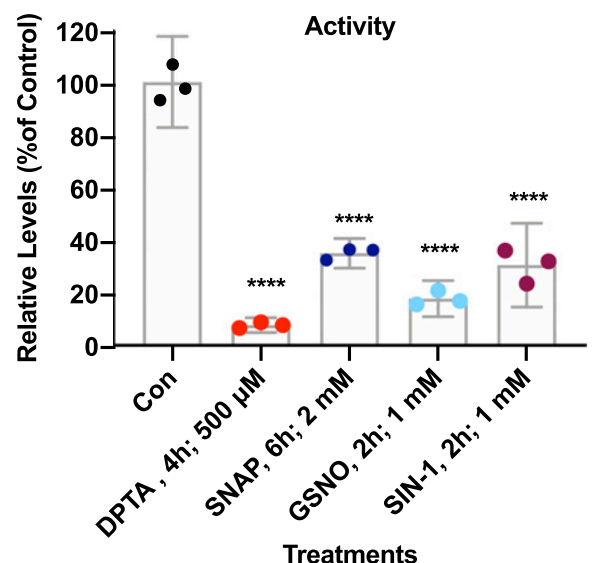

C

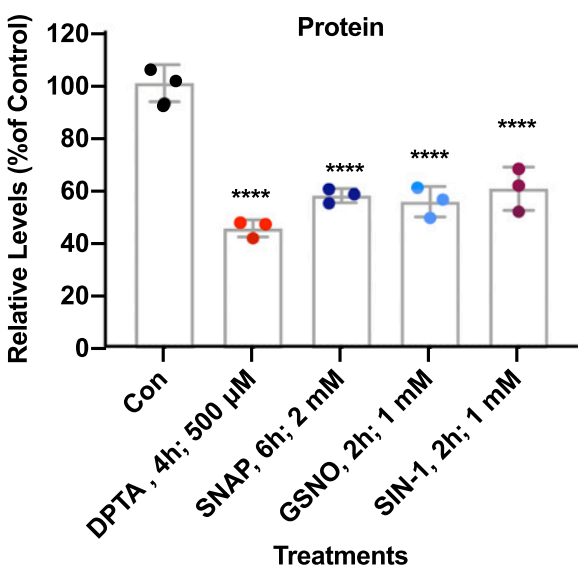

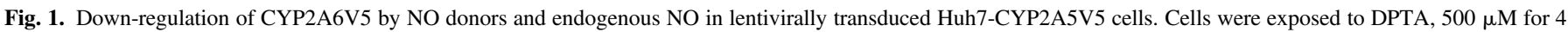

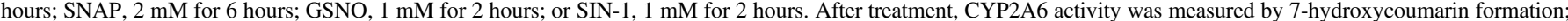

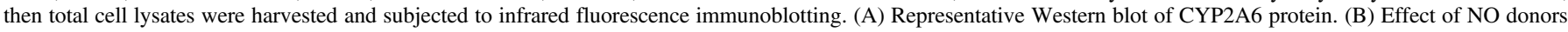

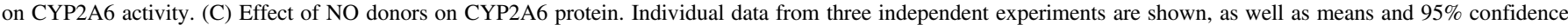

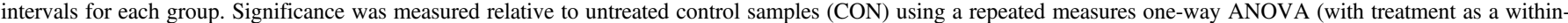
subjects factor) and Dunnett's test. $* * * * P<.0001$. MW, molecular weight.

to cellular function (Aitken et al., 2008; Lee et al., 2008, 2017; Park et al., 2018). Cytochrome P450 2A6 (CYP2A6), primarily located in liver and lung, metabolizes nearly $80 \%$ of nicotine to cotinine (Raunio and Rahnasto-Rilla, 2012). CYP2A6 also metabolizes a number of anesthetics and carcinogens and also coumarin, a substrate used to identify its enzymatic activity (Smith et al., 2007; Raunio and RahnastoRilla, 2012). Furthermore, CYP2A6 activity has been implicated in cigarette consumption rate and probability of becoming a chronic smoker, with low nicotine metabolizers smoking less intensively to achieve the same effective nicotine dosage compared with individuals who metabolize nicotine more rapidly (Tyndale et al., 1999; Tyndale and Sellers, 2002; Park et al., 2017b; Perez-Rubio et al., 2017). Additionally, reduced CYP2A6 activity may result in reduced activation of the lung carcinogen 4-(methylnitrosamino)-1-(3-pyridyl)-1-butanone by CYP2A6, which would lead to lower levels of 4-(methylnitrosamino)1-(3-pyridyl)-1-butanone-related DNA adducts and thus a potential decrease in tumorigenesis (Park et al., 2017b).

Several different $\mathrm{P} 450$ proteins are known to be down-regulated by NO, including CYP2C22, CYP2B1, CYP2B6, CYP2J2, and CYP51A1; however, the pathway of this down-regulation and degradation is variable among different P450s (Lee et al., 2008, 2014, 2017; Park et al., 2017a, 2018). Contrarily, not all $\mathrm{P} 450$ proteins are sensitive to NOdependent down-regulation, including CYP2C11 and CYP3A4 (Chen et al., 1995; Aitken et al., 2008; Lee et al., 2017). Protein degradation occurs via two main pathways: the proteasome complex and the lysosome. Previous studies with CYP2B1 show that induction of nitric oxide synthase 2 resulted in NO-dependent degradation of CYP2B1 via the proteasome in a ubiquitin-dependent manner (Lee et al., 2008). NOdependent degradation of different $\mathrm{P} 450$ proteins has also been observed to occur via multiple pathways. CYP51A1 was observed to be degraded through a combination of proteasomal and calpain pathways (Park et al., 2017a). Similar studies with CYP2C22 have shown that NO-dependent degradation was not attenuated by proteasomal, lysosomal or calpain inhibitors, suggesting that a novel proteolytic system may be implicated (Lee et al., 2014).

This study aims to investigate a posttranslational regulatory pathway of CYP2A6 protein level and its activity via NO-dependent downregulation. Furthermore, we showed that a NO scavenger species, PTIO (carboxy PTIO; 2-(4-carboxyphenyl)-4,5-dihydro-4,4,5,5-tetramethyl1H-imidazolyl-1-oxy-3-oxide, monopotassium salt), can attenuate the degradation of CYP2A6 in a dose-dependent manner. This study provides relevant information regarding the modulation of CYP2A6 enzymatic activity and thus may offer insight on nicotine metabolism and its ramifications in nicotine addiction.

\section{Materials and Methods}

Reagents. Fetal bovine serum was purchased from Atlanta Biologicals (Flowery Branch, GA). Dulbecco's modified Eagle's medium (DMEM) was from Corning (Corning, NY). MycoZap Plus-PR was from Lonza (Morristown, NJ). Penicillin/streptomycin and $0.25 \%$ trypsin EDTA were purchased from Gibco (Waltham, MA). Dipropylenetriamine NONOate (DPTA) and carbobenzoxy-L-leucyl-L-leucyl-L-leucinal (MG132) were purchased from Cayman Chemicals (Ann Arbor, MI). ((2S,3S)-trans-Epoxysuccinyl-L-leucylamido-3methylbutane ethyl ester (E64d, also known as EST) was obtained from Calbiochem (San Diego, CA). PTIO was purchased from Enzo Life Sciences (Farmingdale, NY). Chloroquine was purchased from Sigma-Aldrich (St. Louis, MO). Bortezomib was purchased from LC Laboratories (Woburn, MA), and 3methyladenine (3MA) was purchased from ACROS Organics (Geel, Belgium). Mouse and rabbit monoclonal antibodies to the V5-peptide (V8012 and V8137, respectively) were purchased from Sigma-Aldrich. Mouse monoclonal antibody to glyceraldehyde-3-phosphate dehydrogenase (GAPDH; MAB374) was purchased 
A

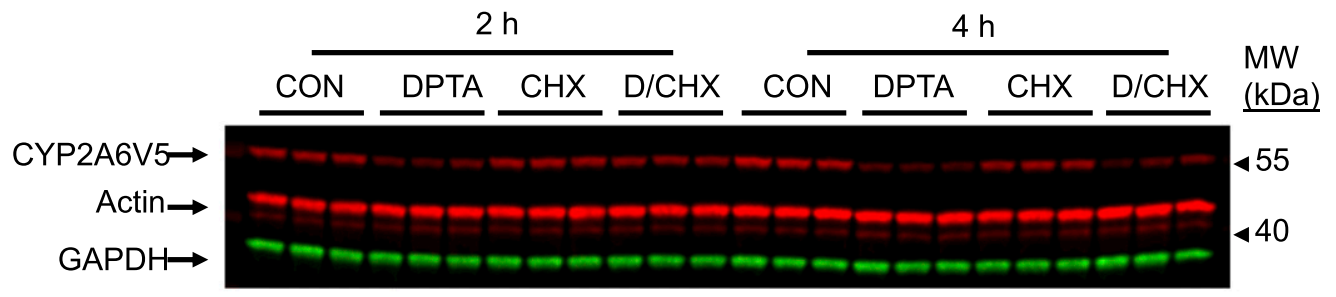

Fig. 2. Posttranslational down-regulation of CYP2A6 in Huh7-CYP2A6V5 cells. Huh7-CYP2A5V5 cells were treated with $500 \mu \mathrm{M}$ DPTA in the presence or absence of $100 \mu \mathrm{g} / \mathrm{ml}$ cycloheximide (CHX) for 2- and 4-hour time periods. (A) Representative Western blot of CHX treatment of 2 and 4 hours. (B) CYP2A6 activity assay. (C) Protein levels were quantified by Western blot analysis. Significance was measured relative to untreated control samples (CON) for each respective time period of 2 or 4 hours. Individual data from three independent experiments are shown, as well as means and $95 \%$ confidence intervals for each group. Data were analyzed by repeated measures two-way ANOVA with time as a within-subjects factor and treatment as a between-subjects factor. $P$ values (Tukey's test) are shown for significant differences. $* * * * P<0.0001$. MW, molecular weight.

from Millipore (Billerica, MA). Affinity purified rabbit anti-actin antibody (A2066) was purchased from Sigma-Aldrich. IRDye 680RD goat anti-rabbit IgG and IRDye 800CW goat anti-mouse IgG were from LI-COR Biosciences (Lincoln, NE). Anti-V5-tag mAb-magnetic beads were obtained from MBL International (Woburn, MA). Rabbit anti-ubiquitin IgG (3933S) was obtained from Cell Signaling Technology (Danvers, MA).

Generation, Culture, and Treatment of Huh7-2A6V5 Cell Line. Human hepatoma Huh7 cell lines expressing CYP2A6 with a C-terminal V5 tag were generated as described for CYP2J2 (Park et al., 2017a). To produce virus particles, HEK293T cells were transfected using a second-generation lentiviral packaging system consisting of pMD2.G and psPAX2 and PLX304-2A6V5 plasmid from the DNASU plasmid repository (Tempe, AZ). Media containing virus were collected after 48 and 72 hours of transfection, filtered through a $0.45 \mu \mathrm{m}$ filter, and stored at $-80^{\circ} \mathrm{C}$. Huh7 cells were infected with $10 \% \mathrm{FBS} / 1 \%$ penicillin/ streptomycin/DMEM containing polybrene $(8 \mu \mathrm{g} / \mathrm{ml})$ to enhance transduction. Infected cells were selected with DMEM culture media containing $10 \mu \mathrm{g} / \mathrm{ml}$ blasticidin. Huh7-2A6V5 cells were cultured in 10\% FBS/1\% penicillin/streptomycin/DMEM at $5 \% \mathrm{CO} 2$ and $37^{\circ} \mathrm{C}$. Cells were grown in 12- or 24-well cell culture plates and treated with indicated condition when the confluence reached $95 \%-100 \%$. Cells were treated with various NO donors and/or drugs as noted in the figure legends. After incubation, the media were removed, and cell lysis buffer was added to the wells. Once the cells were fully digested, the total cell lysates were collected and centrifuged at $12,000 \mathrm{~g}$ for 5 minutes. The supernatant was collected and prepared for SDS-PAGE.

SDS-PAGE and Western Blot Assay. Cells at 95\%-100\% confluence were harvested with cell lysis buffer containing $50 \mathrm{mM}$ Tris-Cl, $\mathrm{pH} 7.5,0.1 \%$ SDS, $1 \%$ NP-40, 1 mM EDTA, and a protease inhibitor cocktail (P8340; SigmaAldrich). Cell lysates were centrifuged at $12,000 \mathrm{~g}$ for 5 minutes, and the supernatants were collected. Total cell lysates were separated by SDS-PAGE on Criterion Stain-Free Precast Gels $(8 \%-16 \%, 5678085)$ from Bio-Rad Laboratories (Hercules, CA) and transferred to nitrocellulose membranes. All antibodies were prepared at the specified dilutions ( $\mathrm{v} / \mathrm{v}$ ) in PBS containing 2\% bovine serum albumin and $0.5 \%$ Tween 20 . The membranes were probed overnight on a rocker at $4^{\circ} \mathrm{C}$ with anti-V5 (1:5000; Sigma) or anti-ubiquitin (1:500), anti-GAPDH (1: 10,000; Millipore), and anti-actin (1:10,000; Sigma) primary antibodies, washed with blotting buffer (PBS/0.05\% Tween20), and secondary anti-mouse and antirabbit antibodies (1:10,000, respectively; LI-COR) were incubated for 1 hour in the dark. After washing blots, the fluorescence signals were visualized by an Odyssey FC imaging system (LI-COR Biosciences). Fluorescence intensity was analyzed using Image Studio software (LI-COR Biosciences). In all experiments with the exception of those depicted in Fig. 1, both GAPDH and actin antibodies were present, and the relative CYP2A6 contents of the samples were normalized to both loading controls. In the experiments depicted in Fig. 1, actin antibody was not employed, and the relative CYP2A6 contents of the samples were normalized to only GAPDH loading controls.

CYP2A6 Activity Assay. Cells were incubated at $37^{\circ} \mathrm{C}$ in $5 \% \mathrm{CO}_{2}$ for 10 minutes in the presence of $\mathrm{P} 450$ assay buffer containing $50 \mu \mathrm{M}$ of coumarin (ACROS Organics), $1 \mathrm{mM} \mathrm{Na}_{2} \mathrm{PO}_{4}, 0.5 \mathrm{mM} \mathrm{MgCl}_{2}, 10 \mathrm{mM}$ HEPES, $5 \mathrm{mM} \mathrm{KCl}$, $10 \mathrm{mM}$ glucose, and $2 \mathrm{mM} \mathrm{CaCl}_{2}$ after removing culture media. After 10 minutes, $60 \mu \mathrm{l}$ of assay media were collected to a 96-well plate, and $100 \mu \mathrm{l} 0.2 \mathrm{M}$ Tris buffer $\mathrm{pH}$ 9.0) was added as a quenching solution. The fluorescence (excitation/ emission, $355 / 460 \mathrm{~nm}$ ) of 7-hydroxycoumarin transformation from coumarin was measured by using FLUOstar Omega plate reader (BMG Labtech).

Ubiquitination Assay. Total cell lysates were collected in the same manner as described in the Western blot assay. Anti-V5 agarose gel beads were prepared by washing with PBS. Total cell lysate supernatants were added to the agarose beads with PBS and a protease inhibitor cocktail (P8340; Sigma-Aldrich) and incubated on a rotary spinner at $4^{\circ} \mathrm{C}$ for 24 hours overnight. The agarose resin was washed five times with $1 \mathrm{ml}$ PBS/1\% NP40 washing buffer. After the final wash, the resins were added to $50 \mu \mathrm{l}$ washing buffer and $50 \mu \mathrm{l}$ of $2 \mathrm{X}$ SDS loading buffer and incubated for 5 minutes at $100^{\circ} \mathrm{C}$, vortexed, and spun before being analyzed by Western blotting.

Data Analyses. Unless otherwise stated, data are presented as the means and 95\% confidence intervals of three independent cell culture experiments. The CYP2A6 levels were normalized with the signals of both actin and GAPDH for each sample. Treatment samples were expressed as a percentage relative to control 


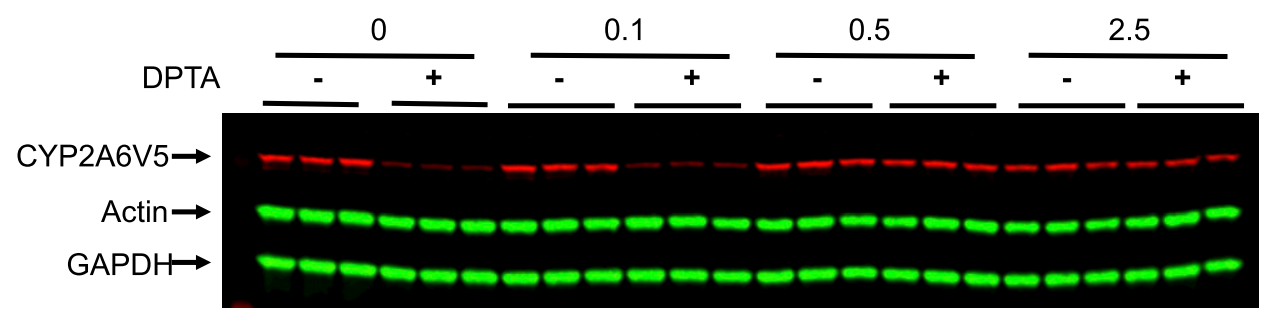

B

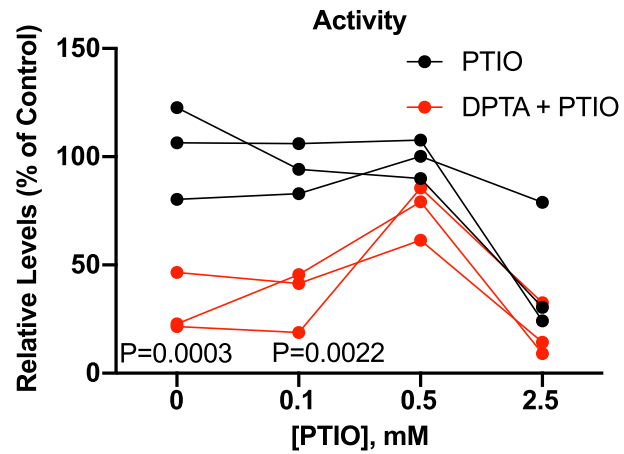

C

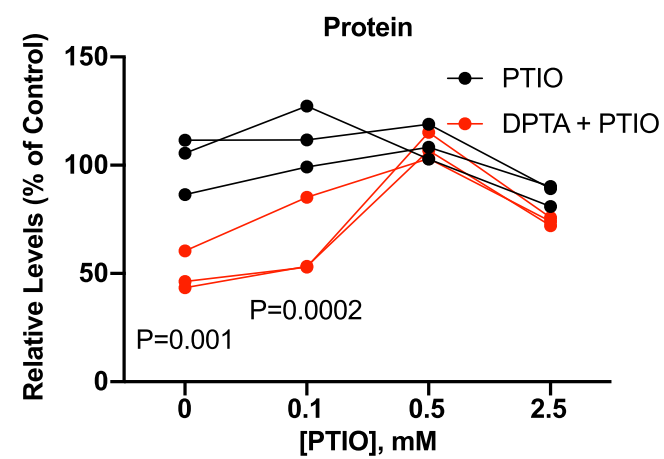

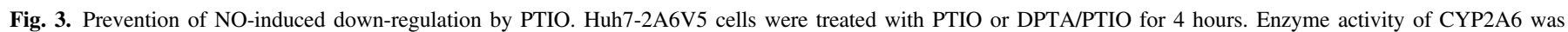

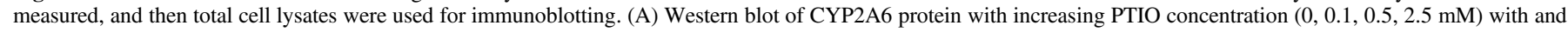

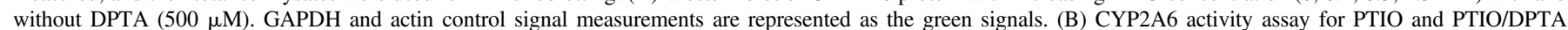

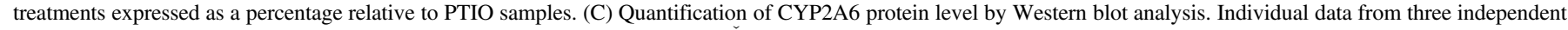

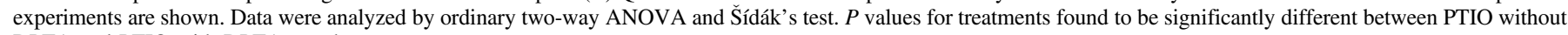
DPTA and PTIO with DPTA are shown.

treatment samples. Statistical analysis and tests were performed using GraphPad Prism 8 (GraphPad Software Inc., La Jolla, CA). Differences among groups were deemed to be significant at $P<0.05$. Details of comparative statistical tests are given in the figure legends, and the outputs from Prism are provided in Supplemental Data.

\section{Results}

Down-Regulation of CYP2A6 by NO Donors. Previous studies demonstrated NO-dependent down-regulation of various P450s by DPTA, a diazeniumdiolate NO donor with a short half-life (3 hours). Therefore, we examined the effects of DPTA and various other NO donors on CYP2A6 down-regulation. 3-Morpholinosydnonimine (SIN1), producing both NO and superoxide spontaneously, is suggested to nitrate tyrosine residues via peroxynitrate production (Rosenkranz et al., 1996). S-nitroso N-acetylpenicillamine (SNAP), an S-nitrosothiol, is commonly used in various biologic applications and may act via trans-Snitrosylation of low molecular weight S-nitrosothiols rather than by releasing free NO (Broniowska and Hogg, 2012). S-nitrosoglutathione (GSNO) is the S-nitrosylated derivative of glutathione, an abundant cellular thiol considered to nitrosylate cysteine residues (Butler and Rhodes, 1997). Thought to exist as a cellular storage system for NO, GSNO/NO levels are shown to modulate autophagy and diseasecausing processes in patients with chronic obstructive pulmonary disease-emphysema (Bodas et al., 2017). Concentrations and times were chosen based on previous experiments (not shown). As seen in Fig. 1B, DPTA and GSNO were the most efficacious in down-regulation of CYP2A6 activity, whereas SNAP and SIN-1 exhibited a significant effect to a lesser observable degree. CYP2A6 protein was down-regulated most effectively by DPTA, whereas GSNO, SNAP, and SIN-1 contributed to the downregulation of protein to a lesser extent (Fig. 1C). In all cases, activities were more affected by the NO donors than were CYP2A6 protein levels, reflecting direct inhibition of the remaining enzyme by NO.

Effect of Protein Synthesis Inhibitor Cycloheximide. To determine whether protein down-regulation occurs posttranslationally, cells were treated in the presence and absence of DPTA and the translational inhibitor cycloheximide for 2 and 4 hours. The extent of down-regulation of CYP2A6 protein and activity in samples treated with DPTA in the presence of cyclohexamide were similar to the observed effect of DPTA-only treatments (Fig. 2). This supports the hypothesis that protein degradation is the main mechanism of down-regulation.

Attenuation of Down-Regulation by PTIO. To establish that the effects of DPTA were due to release of NO, we examined the effect of a nitric oxide scavenger, PTIO, on the down-regulation of CYP2A6V5. We measured both protein level and activity under the treatments of DPTA or DPTA/PTIO for 4 hours (Fig. 3). NO scavenger PTIO effectively inhibited the DPTA-induced down-regulation of CYP2A6 protein and activity levels (Fig. 3), demonstrating that CYP2A6 downregulation by DPTA is NO-dependent. The highest concentration of PTIO itself reduced CYP2A6V5 activity and expression, which was accompanied by observable cell death due to toxicity.

Effects of Protease Inhibitors on Down-Regulation of CYP2A6 by NO. Our previous studies on various cytochrome P450s including CYP2B6, CYP2J2, CYP2B1, and CYP51A1 showed that some cytochrome $\mathrm{P} 450$ enzymes undergo proteasomal degradation in response to NO (Lee et al., 2008, 2017; Park et al., 2017a, 2018). We examined the effect of bortezomib and MG132 (proteasome inhibitors), chloroquine and 3MA (autophagy inhibitors), and E64d (lysosomal and calpain inhibitor) on NO-stimulated CYP2A6 degradation by the NO donor DPTA. We again found a NO-induced reduction in levels of the parent CYP2A6 species by approximately $40 \%$ relative to control (Fig. 4). However, we did not observe a significant effect of the various inhibitors MG132, bortezomib, 3MA, E64d, and chloroquine on NO- 
A

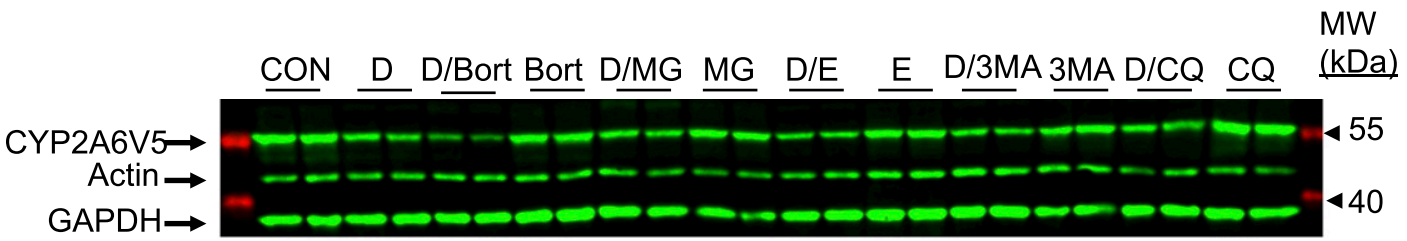

B

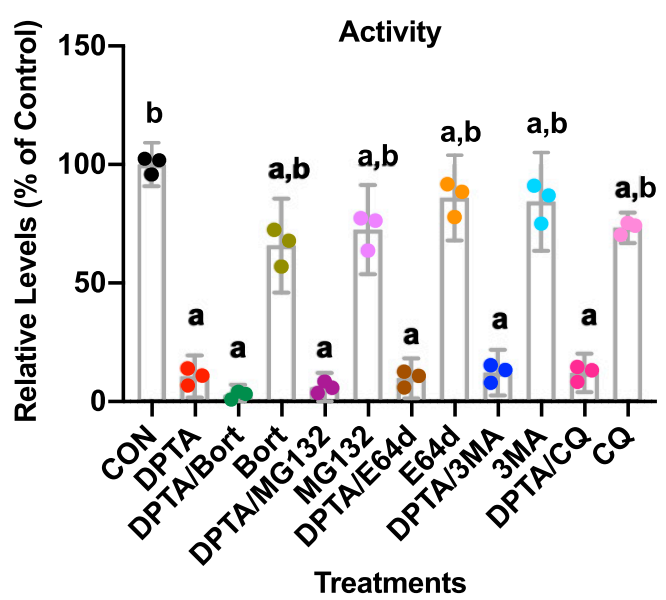

D
C

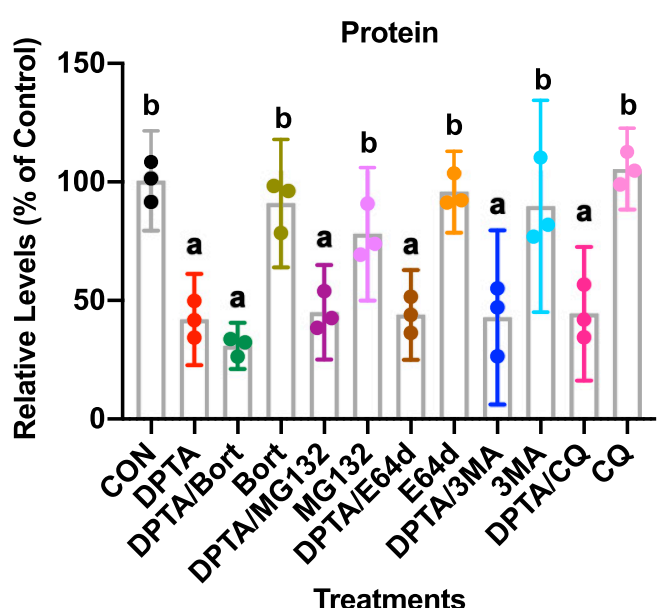

Fig. 4. The effect of protease inhibitors on NO-induced down-regulation of CYP2A6V5. (A) Western blot of CYP2A6 protein exposed for 4 hours to various treatment conditions of $500 \mu \mathrm{M}$ DPTA (D), $10 \mu \mathrm{M}$ bortezomib (Bort), $20 \mu \mathrm{M}$ MG132 (MG), $10 \mu \mathrm{M}$ E64d (E), $10 \mathrm{mM}$ 3MA, and $100 \mu \mathrm{M}$ chloroquine (CQ). (B) Quantification of CYP2A6 activity. (C) Quantification of CYP2A6 protein. Protein level is expressed as a percentage relative to control protein levels (CON). Individual data from three independent experiments are shown, as well as means and 95\% confidence intervals for each group. Repeated measures one-way ANOVA (with treatment as a withinsubjects factor) and Š́dák's test were used to test for differences among treatment groups. a, significantly different from control cells; b, significantly different from cells treated with DPTA alone. $P<0.05$. (D) Western blot detection of a high molecular mass signal by increasing the gain on the fluorescence intensity of (A). Results are the means \pm S.D. of three independent experiments. MW, molecular weight.

induced down-regulation of CYP2A6 protein levels (Fig. 4, A and C). Upon increasing the intensity of the infrared fluorescence signal, the accumulation of high molecular mass (HMM) species containing CYP2A6 became apparent under the cotreatment of the proteasome inhibitors with DPTA (Fig. 4D). This led us to the hypothesis that the HMM species could be polyubiquitinated CYP2A6.

Enhancement of High Molecular Mass Species by NO Donor and Proteasome Inhibitor Cotreatments. To substantiate and quantify the formation of HMM species by the cotreatment of DPTA and proteasome inhibitors, we designed an experiment with only bortezomib and MG132 proteasome inhibitors with and without exposure to DPTA. Again, DPTA treatments effectively down-regulated parental CYP2A6 protein and activities (Fig. 5, A-C). Although not statistically significant, the cotreatment of DPTA with proteasome inhibitors MG132 or bortezomib tended to further reduce levels of CYP2A6V5 below those of DPTA treatment alone (Fig. 5, B and C). The HMM species were again observed upon increasing the gain on the fluorescence intensity (Fig. 5A). We quantified the HMM species and found a significant increase with DPTA/proteasome inhibitor treatment compared with DPTA-only treatment (Fig. 5D).

Ubiquitination of CYP2A6. To determine if CYP2A6 is ubiquitinated in response to DPTA treatment and the HMM observed is in fact
CYP2A6 polyubiquitination, we immunoprecipitated the CYP2A6V5 protein from treated cells and examined its ubiquitination state. When we treated Huh7-CYP2A6V5 cells with DPTA, DPTA/MG132, or MG132 followed by immunoprecipitation and probed the Western blot with rabbit anti-V5 antibody, we again observed the presence of HMM species in both the total cell lysate and the immunoprecipitated fraction (Fig. 6A). When the immunoprecipitation was followed by immunoblotting with anti-ubiquitin, we observed a high intensity signal of the polyubiquitinated CYP2A6 complex (Fig. 6B) in the presence of MG132, which was greatly accentuated by DPTA cotreatment (Fig. 6B).

Effect of CYP2A6 Inhibitors on Its NO-Dependent Degradation. We reported previously that the NO-stimulated degradation of CYP2J2 was blocked by its inhibitor danazol (Park et al., 2018). We have also found that inhibitors of CYP2B6 and CYP51A1 block their degradation (Lee et al., unpublished data). Therefore we analyzed the effect of pilocarpine, a mixed competitive and noncompetitive CYP2A6 inhibitor (DeVore et al., 2012) on the down-regulation of CYP2A6V5 by DPTA. CYP2A6 activity in Huh7-CYP2A6V5 cells was inhibited in a dosedependent manner by pilocarpine with an $\mathrm{EC}_{50}$ of approximately $10 \mu \mathrm{M}$. Pilocarpine also blocked the down-regulation of CYP2A6V5 protein with similar potency (Fig. 7, A and C). We then asked whether methoxsalen, a mechanism-based inhibitor of CYP2A6, would have the 
A

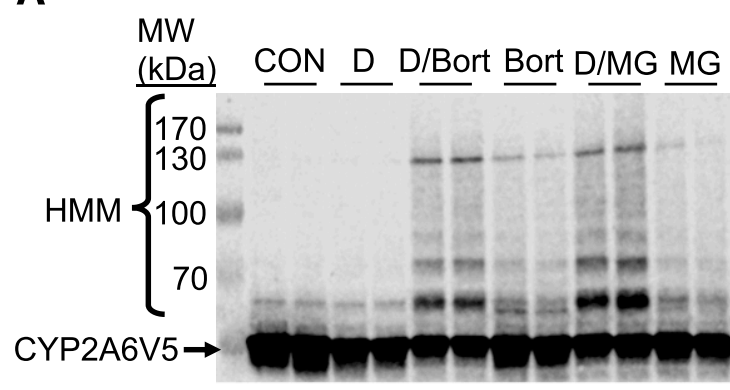

C

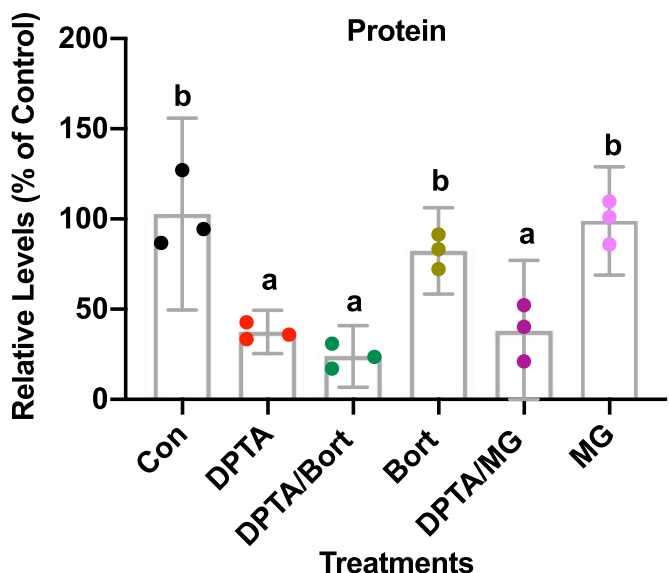

B

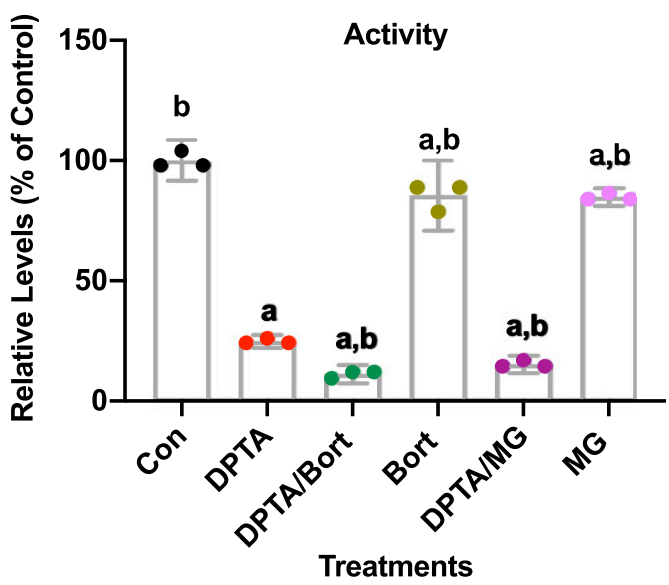

D

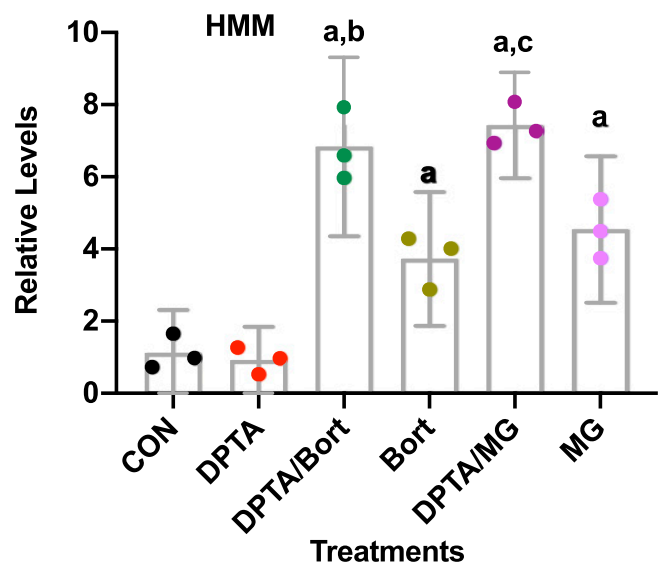

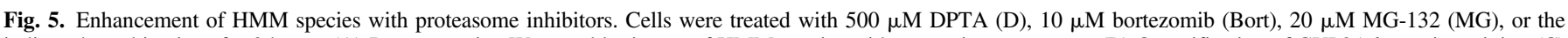

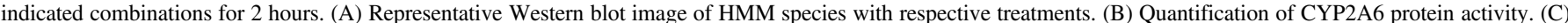

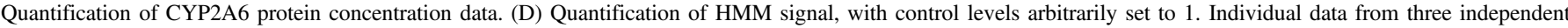

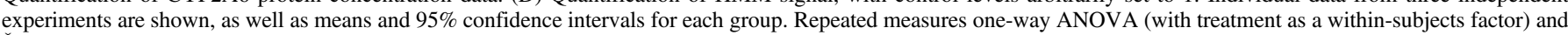

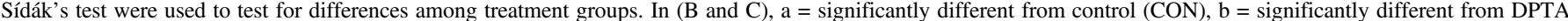

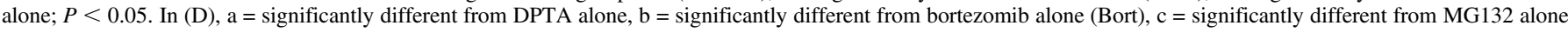
(MG); $P<0.05$. MW, molecular weight.

same effect. Treatment of cells with methoxsalen starting 30 minutes before DPTA addition effectively inhibited CYP2A6 activity with an $\mathrm{EC}_{50}$ of $<0.1 \mu \mathrm{M}$ (Fig. $8 \mathrm{~B}$ ). However, methoxsalen did not protect CYP2A6V5 from DPTA-stimulated degradation at any concentration (Fig. 8, A and C). We verified that 30 minutes of preincubation with methoxsalen was sufficient to inactivate the enzyme and that the inactivation was not reversible by washing the cells prior to measuring activity (Supplemental Fig. 1).

\section{Discussion}

We demonstrated that CYP2A6 protein is down-regulated by various chemical NO donors. We confirmed the down-regulation was in fact by released NO and not the DPTA chemical by using a nitric oxide scavenger species, PTIO. Furthermore, we identified this downregulation to act via a posttranslational pathway and confirmed that CYP2A6 protein is degraded via the ubiquitin-proteasomal pathway.

In previous studies, CYP2J2, CYP2B6, CYP2B1, CYP51A1, and CYP2C22 were identified as targets for NO-induced down-regulation in Huh7 cells or rat primary hepatocytes, respectively (Lee et al., 2008, 2014, 2017; Park et al., 2017a, 2018). CYP2B6 degradation was blocked by proteasome inhibitors, whereas CYP2C22 and CYP2J2 degradation proceed via novel pathways. Thus, different $\mathrm{P} 450$ enzymes are degraded in a NO-dependent manner via diverse pathways. We confirmed that the observed down-regulation of CYP2A6 was in fact induced by NO by using PTIO, a nitric oxide scavenger species. Both activity and protein levels were nearly completely restored to control sample levels when cells were treated with an effective concentration of PTIO. An apparent decrease in activity with $2.5 \mathrm{mM}$ PTIO treatment was observed due to cell toxicity. Although the disappearance of NO-induced CYP2A6 parent protein was not attenuated by proteasome inhibitors, the observed accumulation of HMM species (Fig. 5) and ubiquitinated CYP2A6 (Fig. 6) in cells cotreated with DPTA and either MG132 or bortezomib indicates that the proteasome inhibitors did indeed attenuate degradation of the ubiquitinated CYP2A6. Furthermore, the fact that ubiquitinated CYP2A6 only accumulated in samples treated with both DPTA and proteasome inhibitor indicates that degradation occurs rapidly following NO-induced ubiquitination. It is notable that the pattern of HMM species detected by the V5 antibodies in Fig. 6A is different from the ubiquitination pattern detected in Fig. 6B. Part of the reason is that the ubiquitination pattern is biased toward multiubiquitinated species that bind multiple ubiquitin antibodies, whereas each CYP2A6 species will only be labeled by one V5 antibody. However, we cannot exclude the possibility that the HMM species also contain CYP2A6 oligomers or aggregates.

CYP2A6 degradative pathways resemble those observed in both CYP51A1 as well as CYP2B6 (Lee et al., 2017; Park et al., 2017a). 

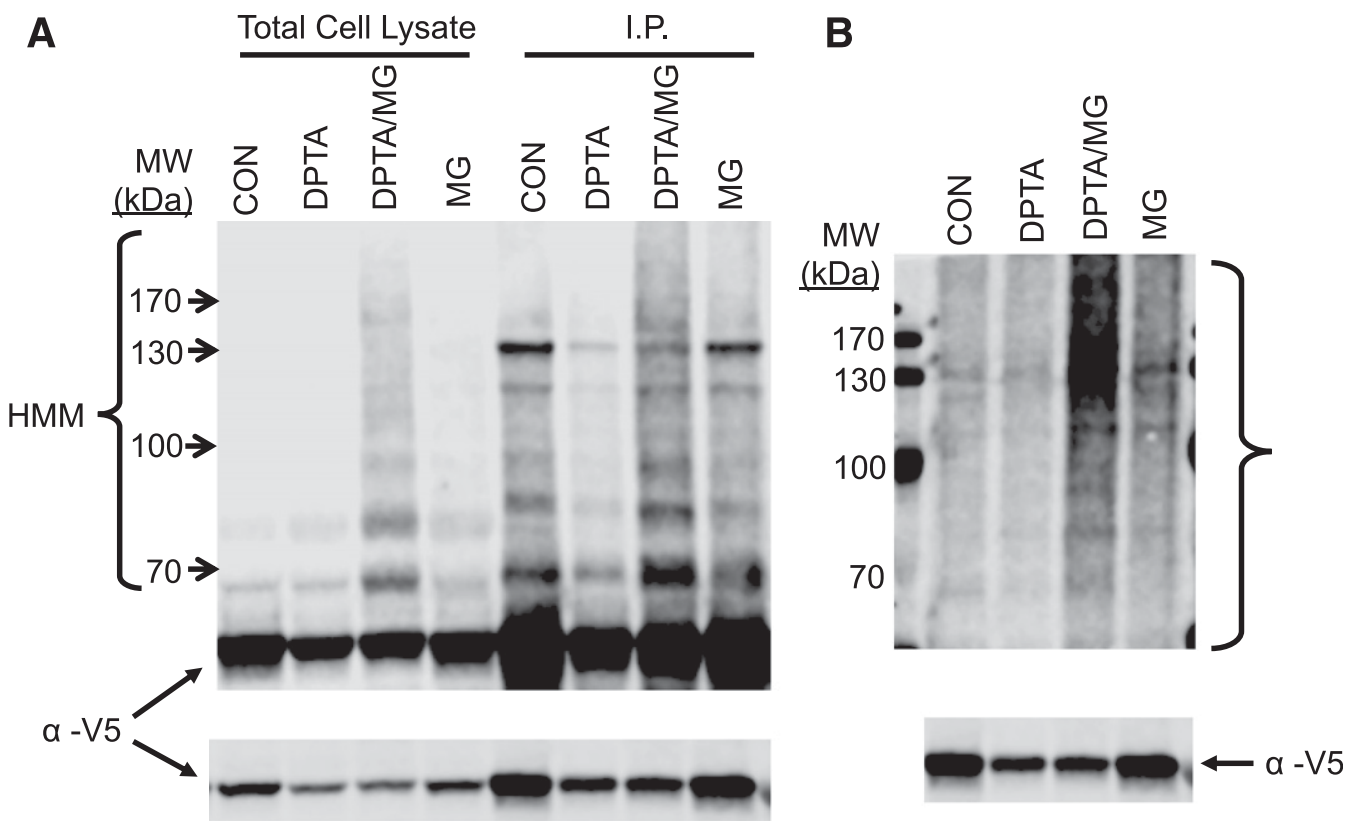

Fig. 6. Enhanced ubiquitination with cotreatment of DPTA and MG132. Cells were treated with either control medium (CON), DPTA (D; $500 \mu M)$, MG132 (MG; $20 \mu M)$, or DPTA + MG132 for 2 hours. Total cell lysates were prepared and subjected to immunoprecipitation with anti-V5 agarose beads (I.P.). (A) The cell lysates and immunoprecipitates were analyzed by Western blotting with anti-V5 antibodies to visualize CYP2A6V5 protein and its corresponding HMM species. (B) Western blot detection of polyubiquitinated CYP2A6. Immunoprecipitates were blotted and probed with anti-ubiquitin antibodies. Representative blots are shown, and the observations were conserved across three independent experiments. MW, molecular weight.

However, for CYP51A1, whereas proteasome inhibitors induced a partial attenuation of protein down-regulation, a lack of HMM complexes in the presence of DPTA and proteasome inhibition suggested alternative pathways of degradation (Park et al., 2017a). In CYP2A6, we were able to confirm that the HMM species were in fact due to CYP2A6 ubiquitination, and we detected high levels of ubiquitination in cells treated with both DPTA and proteasome inhibitors. This is akin to CYP2B6 protein degradation, as cotreatments of DPTA and proteasome inhibitor resulted in the accumulation of HMM species, which was identified as ubiquitinated protein (Lee et al., 2017).

A

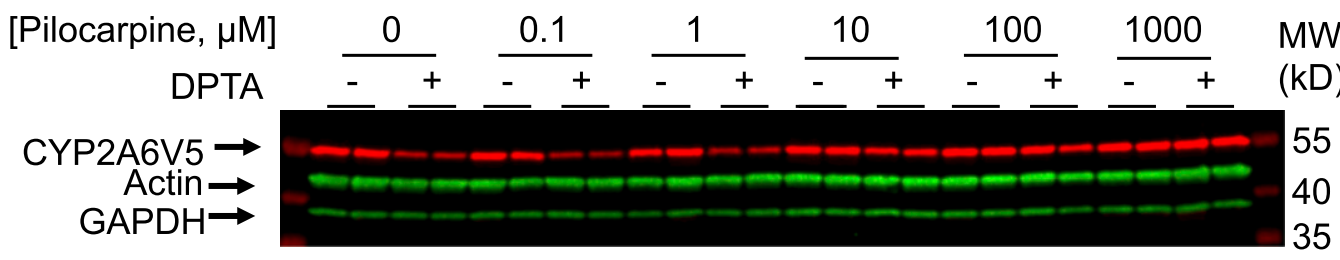

B

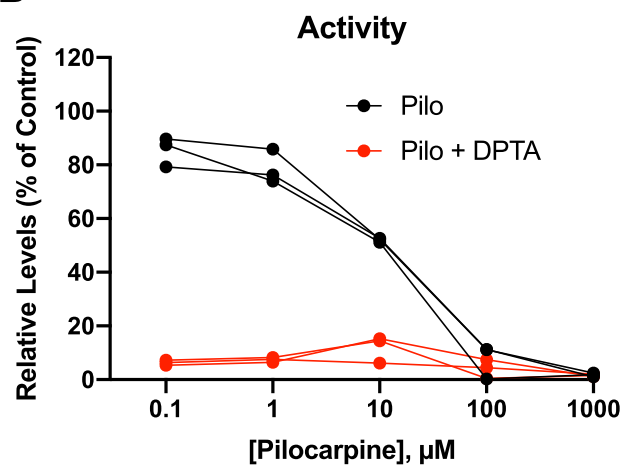

C

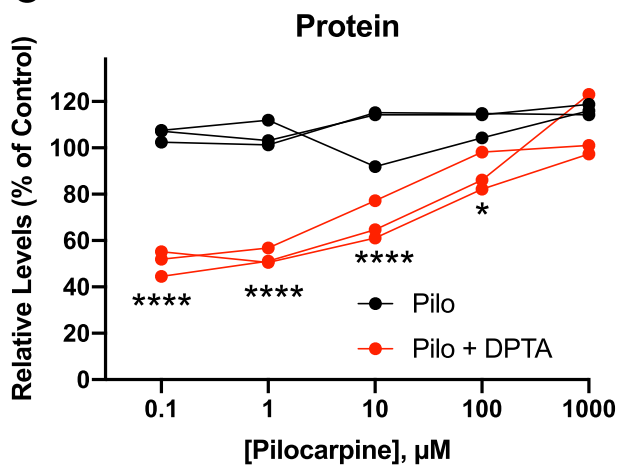

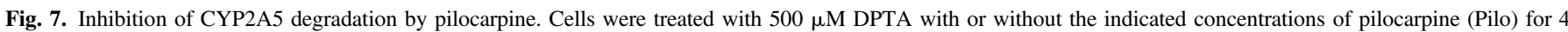

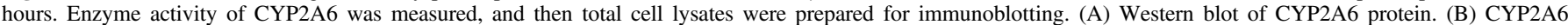

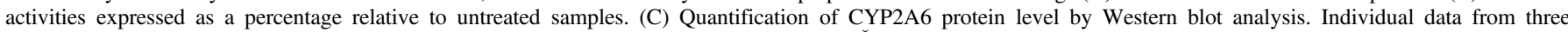

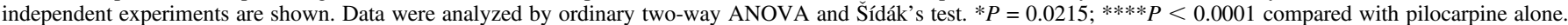
MW, molecular weight. 
A

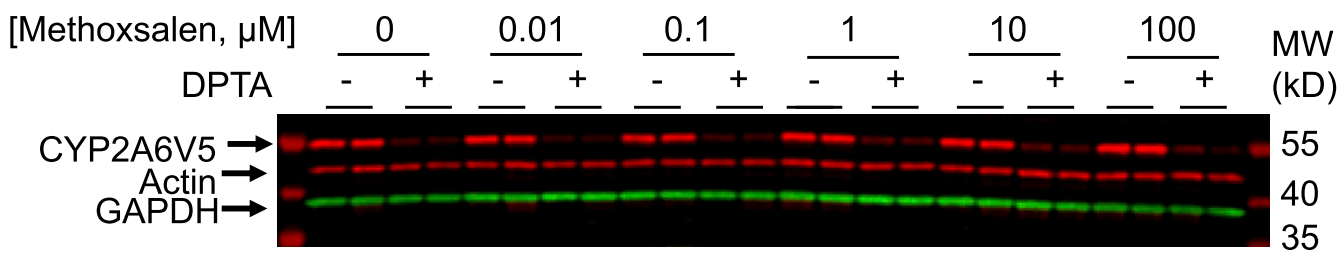

B

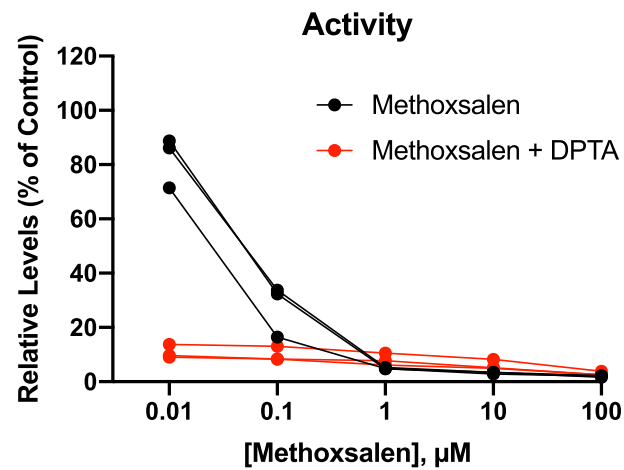

C

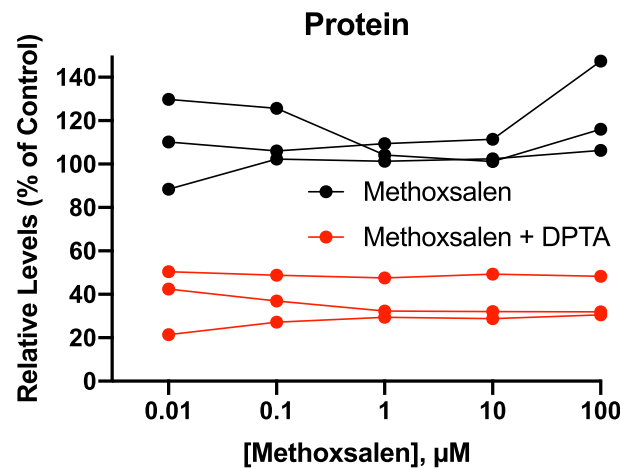

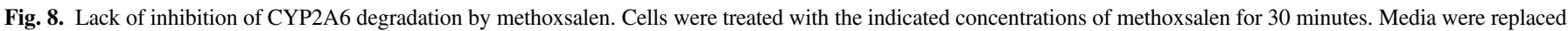

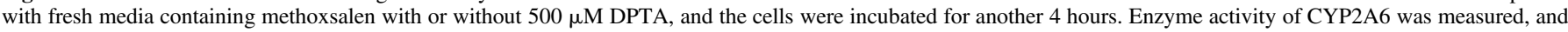

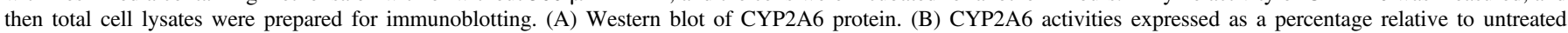

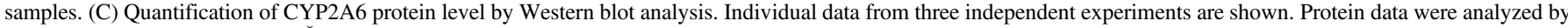

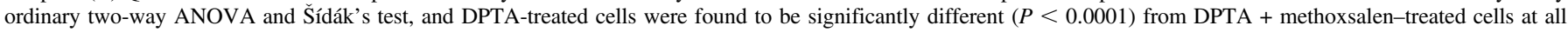
methoxsalen concentrations. MW, molecular weight.

NO is known to react with oxygen and reactive oxygen species to form reactive nitrogen species that can modify proteins to regulate their function or expression (Cooper et al., 2002; Lancaster, 2008; Adams et al., 2015). Most experiments in this study were performed using the NO donor DPTA. The concentration of DPTA found to be effective $(250-500 \mu \mathrm{M})$ is comparable to the concentration of NO necessary to evoke similar reactions of down-regulation in other $\mathrm{P} 450$ proteins exhibiting a similar response to CYP2A6 (Lee et al., 2014, 2017; Park et al., 2017a, 2018). However, the mechanisms by which NO simulates the degradation of these enzymes are unclear. The failure of the mechanism based inhibitor methoxsalen to block the down-regulation of the enzyme (Fig. 8) leads us to conclude that enzymatic activity is not required for down-regulation. This is in contrast to the known mechanism of inactivation of CYP8A by peroxynitrite, which involves heme thiolate-catalyzed tyrosine nitration (Zou et al., 2000). On the other hand, the fact that pilocarpine inhibited CYP2A6 down-regulation with a similar potency to its inhibition of enzyme activity (Fig. 7) suggests that NO needs access to the active site to trigger the degradation process. This could be via interaction with the heme itself, with the axial thiolate ligand, or with other residues in the substrate binding site. Methoxsalen inactivates CYP2A6 by covalent protein modification via a $\gamma$-ketenal reactive metabolite (Koenigs and Trager, 1998), although the amino acids modified are not known. Although methoxsalen and pilocarpine exhibit highly similar binding modes when crystallized with CYP2A6 (Yano et al., 2005; DeVore et al., 2012), the binding affinity of methoxsalen for CYP2A6 is 3-fold lower than that of pilocarpine (Stephens et al., 2012). This difference is likely due to the ligation of the unsubstituted nitrogen in pilocarpine's imidazole ring to CYP2A6 (DeVore et al., 2012), which generates the characteristic type II spectral shift. The lower affinity of methoxsalen (which does not have a nitrogen atom), in addition to an altered conformation of the substrate binding pocket in the inactivated enzyme, likely explains its inability to block down-regulation via competitive binding.

Nitric oxide-induced degradation has been documented in proteins beyond the P450 family. Cytoglobin, a redox-regulatory protein involved in cellular protection against oxidative stress was found to undergo heme nitrosylation when exposed to reactive nitrogen species, resulting in conformational changes (De Backer et al., 2018). Additionally, reactive nitrogen species induced protein degradation of nuclear factor- $\kappa \mathrm{B}$, whose improper regulation has been linked to many disease states including cancer, autoimmune diseases, and viral or bacterial infection (Bar-Shai and Reznick, 2006). Future studies aim to further elucidate the mechanism by which NO interacts with CYP2A6 protein.

In conclusion, NO elicits down-regulation of CYP2A6 via ubiquitination and proteasomal degradation. CYP2A6 is involved in the metabolism of several xenobiotics including nicotine, and its regulation by NO can cause adverse drug interactions or abnormal nicotine metabolism. Previous studies have found that inhaled NO from cigarette smoke as well as NO released by nicotine may contribute to the development of nicotine addiction (Vleeming et al., 2002). CYP2A6 is responsible for nicotine metabolism, and its regulation by NO could lead to further implications on nicotine metabolism and addiction.

\section{Authorship Contributions \\ Participated in research design: Cerrone, Lee, Mi, Morgan. \\ Conducted experiments: Cerrone, Lee, Mi. \\ Performed data analysis: Cerrone, Lee, Mi, Morgan. \\ Wrote or contributed to the writing of the manuscript: Cerrone, Lee, Morgan.}

\section{References}

Adams L, Franco MC, and Estevez AG (2015) Reactive nitrogen species in cellular signaling. Exp Biol Med (Maywood) 240:711-717.

Agvald P, Adding LC, Artlich A, Persson MG, and Gustafsson LE (2002) Mechanisms of nitric oxide generation from nitroglycerin and endogenous sources during hypoxia in vivo. $\mathrm{Br}$ J Pharmacol 135:373-382. 
Aitken AE, Lee CM, and Morgan ET (2008) Roles of nitric oxide in inflammatory downregulation of human cytochromes P450. Free Radic Biol Med 44:1161-1168.

Antoniades C, Bakogiannis C, Leeson P, Guzik TJ, Zhang MH, Tousoulis D, Antonopoulos AS Demosthenous M, Marinou K, Hale A, et al. (2011) Rapid, direct effects of statin treatment on arterial redox state and nitric oxide bioavailability in human atherosclerosis via tetrahydrobiopterin-mediated endothelial nitric oxide synthase coupling. Circulation 124: $335-345$.

Arnold WP, Mittal CK, Katsuki S, and Murad F (1977) Nitric oxide activates guanylate cyclase and increases guanosine $3^{\prime}: 5^{\prime}$-cyclic monophosphate levels in various tissue preparations. Proc Nat Acad Sci USA 74:3203-3207.

Bar-Shai M and Reznick AZ (2006) Reactive nitrogen species induce nuclear factor-kappaBmediated protein degradation in skeletal muscle cells. Free Radic Biol Med 40:2112-2125.

Bartesaghi S, Herrera D, Martinez DM, Petruk A, Demicheli V, Trujillo M, Martí MA, Estrín DA, and Radi R (2017) Tyrosine oxidation and nitration in transmembrane peptides is connected to lipid peroxidation. Arch Biochem Biophys 622:9-25.

Bartesaghi S and Radi R (2018) Fundamentals on the biochemistry of peroxynitrite and protein tyrosine nitration. Redox Biol 14:618-625.

Beuve A (2017) Thiol-based redox modulation of soluble guanylyl cyclase, the nitric oxide receptor. Antioxid Redox Signal 26:137-149.

Bodas M, Silverberg D, Walworth K, Brucia K, and Vij N (2017) Augmentation of S-nitrosoglutathione controls cigarette smoke-induced inflammatory-oxidative stress and chronic obstructive pulmonary disease-emphysema pathogenesis by restoring cystic fibrosis transmembrane conductance regulator function. Antioxid Redox Signal 27:433-451.

Broniowska KA and Hogg N (2012) The chemical biology of S-nitrosothiols. Antioxid Redox Signal 17:969-980.

Butler AR and Rhodes P (1997) Chemistry, analysis, and biological roles of S-nitrosothiols. Anal Biochem 249:1-9.

Chakravarti B and Chakravarti DN (2017) Protein tyrosine nitration: role in aging. Curr Aging Sci 10:246-262.

Chen J, Nikolova-Karakashian M, Merrill AH Jr., and Morgan ET (1995) Regulation of cytochrome P450 2C11 (CYP2C11) gene expression by interleukin-1, sphingomyelin hydrolysis, and ceramides in rat hepatocytes. J Biol Chem 270:25233-25238.

Cooper CE, Patel RP, Brookes PS, and Darley-Usmar VM (2002) Nanotransducers in cellular redox signaling: modification of thiols by reactive oxygen and nitrogen species. Trends Biochem Sci 27:489-492.

De Backer J, Razzokov J, Hammerschmid D, Mensch C, Hafideddine Z, Kumar N, van Raemdonck G, Yusupov M, Van Doorslaer S, Johannessen C, et al. (2018) The effect of reactive oxygen and nitrogen species on the structure of cytoglobin: a potential tumor suppressor. Redox Biol 19:1-10.

DeVore NM, Meneely KM, Bart AG, Stephens ES, Battaile KP, and Scott EE (2012) Structural comparison of cytochromes P450 2A6, 2A13, and 2E1 with pilocarpine. FEBS J 279:1621-1631.

Hess DT and Stamler JS (2012) Regulation by S-nitrosylation of protein post-translational modification. $J$ Biol Chem 287:4411-4418.

Hunt AP and Lehnert N (2015) Heme-nitrosyls: electronic structure implications for function in biology. Acc Chem Res 48:2117-2125.

Kim S, Wing SS, and Ponka P (2004) S-nitrosylation of IRP2 regulates its stability via the ubiquitin-proteasome pathway. Mol Cell Biol 24:330-337.

Koenigs LL and Trager WF (1998) Mechanism-based inactivation of P450 2A6 by furanocoumarins. Biochemistry 37:10047-10061.

Lancaster JR Jr. (2008) Protein cysteine thiol nitrosation: maker or marker of reactive nitrogen species-induced nonerythroid cellular signaling? Nitric Oxide 19:68-72.

Laufs U and Liao JK (1998) Post-transcriptional regulation of endothelial nitric oxide synthase mRNA stability by Rho GTPase. J Biol Chem 273:24266-24271.

Lee CM, Kim BY, Li L, and Morgan ET (2008) Nitric oxide-dependent proteasomal degradation of cytochrome P450 2B proteins. J Biol Chem 283:889-898.

Lee CM, Lee BS, Arnold SL, Isoherranen N, and Morgan ET (2014) Nitric oxide and interleukin$1 \beta$ stimulate the proteasome-independent degradation of the retinoic acid hydroxylase CYP2C 22 in primary rat hepatocytes. J Pharmacol Exp Ther 348:141-152.

Lee CM, Tripathi S, and Morgan ET (2017) Nitric oxide-regulated proteolysis of human CYP2B6 via the ubiquitin-proteasome system. Free Radic Biol Med 108:478-486.

Mylonas C and Kouretas D (1999) Lipid peroxidation and tissue damage. In Vivo 13:295-309.
Nakamura T, Prikhodko OA, Pirie E, Nagar S, Akhtar MW, Oh CK, McKercher SR, Ambasudhan R, Okamoto S, and Lipton SA (2015) Aberrant protein S-nitrosylation contributes to the pathophysiology of neurodegenerative diseases. Neurobiol Dis 84:99-108.

Park JW, Byrd A, Lee CM, and Morgan ET (2017a) Nitric oxide stimulates cellular degradation of human CYP51A1, the highly conserved lanosterol 14 $\alpha$-demethylase. Biochem J 474:3241-3252.

Park JW, Lee CM, Cheng JS, and Morgan ET (2018) Posttranslational regulation of CYP2J2 by nitric oxide. Free Radic Biol Med 121:149-156.

Park SL, Murphy SE, Wilkens LR, Stram DO, Hecht SS, and Le Marchand L (2017b) Association of CYP2A6 activity with lung cancer incidence in smokers: the multiethnic cohort study. PLoS One 12: 0178435.

Pérez-Rubio G, López-Flores LA, Ramírez-Venegas A, Noé-Díaz V, García-Gómez L, AmbrocioOrtiz E, Sánchez-Romero C, Hernández-Zenteno RJ, Sansores RH, and Falfán-Valencia R (2017) Genetic polymorphisms in CYP2A6 are associated with a risk of cigarette smoking and predispose to smoking at younger ages. Gene 628:205-210.

Radi R (2013) Protein tyrosine nitration: biochemical mechanisms and structural basis of functional effects. Acc Chem Res 46:550-559.

Radi R (2018) Oxygen radicals, nitric oxide, and peroxynitrite: redox pathways in molecular medicine. Proc Natl Acad Sci USA 115:5839-5848.

Raunio H and Rahnasto-Rilla M (2012) CYP2A6: genetics, structure, regulation, and function. Drug Metabol Drug Interact 27:73-88.

Rosenkranz B, Winkelmann BR, and Parnham MJ (1996) Clinical pharmacokinetics of molsidomine. Clin Pharmacokinet 30:372-384

Sayed N, Baskaran P, Ma X, van den Akker F, and Beuve A (2007) Desensitization of soluble guanylyl cyclase, the NO receptor, by S-nitrosylation. Proc Natl Acad Sci USA 104: 12312-12317.

Shah RC, Sanker S, Wood KC, Durgin BG, and Straub AC (2018) Redox regulation of soluble guanylyl cyclase. Nitric Oxide 76:97-104.

Smith BD, Sanders JL, Porubsky PR, Lushington GH, Stout CD, and Scott EE (2007) Structure of the human lung cytochrome P450 2A13. J Biol Chem 282:17306-17313.

Stephens ES, Walsh AA, and Scott EE (2012) Evaluation of inhibition selectivity for human cytochrome P450 2A enzymes. Drug Metab Dispos 40:1797-1802.

Tyndale RF, Pianezza ML, and Sellers EM (1999) A common genetic defect in nicotine metabolism decreases risk for dependence and lowers cigarette consumption. Nicotine Tob Res 1 (Suppl 2):S63-S67, NaN-S70.

Tyndale RF and Sellers EM (2002) Genetic variation in CYP2A6-mediated nicotine metabolism alters smoking behavior. Ther Drug Monit 24:163-171.

Vleeming W, Rambali B, and Opperhuizen A (2002) The role of nitric oxide in cigarette smoking and nicotine addiction. Nicotine Tob Res 4:341-348.

Yano JK, Hsu MH, Griffin KJ, Stout CD, and Johnson EF (2005) Structures of human microsomal cytochrome P450 2A6 complexed with coumarin and methoxsalen. Nat Struct Mol Biol 12: 822-823.

Yeo WS, Kim YJ, Kabir MH, Kang JW, Ahsan-Ul-Bari M, and Kim KP (2015) Mass spectrometric analysis of protein tyrosine nitration in aging and neurodegenerative diseases. Mass Spectrom Rev 34:166-183.

Zahid S, Khan R, Oellerich M, Ahmed N, and Asif AR (2014) Differential S-nitrosylation of proteins in Alzheimer's disease. Neuroscience 256:126-136.

Zhan X, Huang Y, and Qian S (2018) Protein tyrosine nitration in lung cancer: current research status and future perspectives. Curr Med Chem 25:3435-3454.

Zhao QF, Yu JT, and Tan L (2015) S-Nitrosylation in Alzheimer's disease. Mol Neurobiol 51 268-280.

Zou MH, Daiber A, Peterson JA, Shoun H, and Ullrich V (2000) Rapid reactions of peroxynitrite with heme-thiolate proteins as the basis for protection of prostacyclin synthase from inactivation by nitration. Arch Biochem Biophys 376:149-155.

Address correspondence to: Dr. Edward T. Morgan, Department of Pharmacology and Chemical Biology, Emory University School of Medicine, 5119 Rollins Research Center, 1510 Clifton Rd., Atlanta, GA 30322. E-mail: edward.morgan@ emory.edu 